\title{
Range-Kernel Orthogonality and Elementary Operators: The Nonsmoothness Case
}

\author{
A. Bachir $\left(\mathbb{D},{ }^{1}\right.$ A. Segres, ${ }^{2}$ and Nawal Sayyaf ${ }^{3}$ \\ ${ }^{1}$ Department of Mathematics, University of King Khalid, Abha, Saudi Arabia \\ ${ }^{2}$ University of Mascara, Mascara, Algeria \\ ${ }^{3}$ Department of Mathematics, College of Science, University of Bisha, Bisha, Saudi Arabia \\ Correspondence should be addressed to A. Bachir; abishr@kku.edu.sa
}

Received 3 December 2019; Accepted 25 May 2020; Published 24 June 2020

Academic Editor: Eulalia Martínez

Copyright (C) 2020 A. Bachir et al. This is an open access article distributed under the Creative Commons Attribution License, which permits unrestricted use, distribution, and reproduction in any medium, provided the original work is properly cited.

The characterization of the points in $C_{1}(\mathscr{H})$, the trace class operators, that are orthogonal to the range of elementary operators has been carried out for certain kinds of elementary operators by many authors in the smooth case. In this note, we study that the characterization is a problem in nonsmoothness case for general elementary operators, and we give a counter example to S. Mecheri and M. Bounkhel results.

\section{Introduction}

Let $B(\mathscr{H})$ be the algebra of all bounded linear operators acting on a complex separable Hilbert space $\mathscr{H}$ and $A \in B(\mathscr{H})$ be compact operator, and let $s_{1}(A) \geq s_{2}(A) \geq \cdots \geq 0$ denote the eigenvalues of $|A|=\left(A^{*} A\right)^{1 / 2}$ arranged in their decreasing order. $A \in C_{1}(\mathscr{H})$, the class trace, if

$$
\|A\|_{1}=\sum_{i=1}^{\infty} s_{i}(A)=\operatorname{tr}|A|<\infty,
$$

where $\operatorname{tr}$ denotes the trace functional [1].

We recall the definition of Birkhoff-James's orthogonality in Banach spaces $[2,3]$.

Definition 1. If $\mathscr{A}$ is a complex Banach space, then for any elements $a, b \in \mathscr{A}$, we say that $a$ is orthogonal to $b$, noted by $a \perp b$, iff for all $\lambda, \beta \in \mathbb{C}$ there holds

$$
\|\lambda b+\beta a\| \geq\|\beta a\|
$$

If $M$ and $N$ are linear subspaces in $\mathscr{A}$, we say that $M$ is orthogonal to $N$, noted by $M \perp N$, if $\|a+b\| \geq\|a\|$ for all $a \in M$ and all $b \in N$. If $M=\operatorname{span}\{a\}$, we simply write $a \perp N$. (i) The orthogonality in this sense is not symmetric

(ii) If $\mathscr{A}$ is a Hilbert space with its inner product $\langle\cdot\rangle$, then it follows from (2) that $\langle a, b\rangle=0$ which means that Birkhoff-James's orthogonality generalizes the usual sense in Hilbert space

We also recall the definition of the range-kernel orthogonality for a pair of operators $(E, T)$ on Banach spaces introduced by R. Harte [4].

Definition 2. If $E: \mathscr{A} \longrightarrow Y$ and $T: \mathscr{B} \longrightarrow \mathscr{C}$ are bounded linear operators between Banach spaces. $T$ is called rangekernel orthogonal to $E$ provided $\operatorname{ker} T$ is orthogonal to $\operatorname{ran} E$ in the sense of the Definition 1, i.e.,

$$
s \in \operatorname{ker} T \Longrightarrow\|s+E(x)\| \geq\|s\|, \quad \text { for all } x \in \mathscr{A} \text {. }
$$

If $T=E$, we shall call that $E$ is orthogonal.

The elementary operator is an operator $E$ defined on Banach $(\mathscr{A}, \mathscr{B})$-bimodule $\mathscr{M}$ with its representation $E(x)=\sum_{i=1}^{n} a_{i} x b_{i}$, where $a=\left(a_{i}\right)_{i} \in \mathscr{A}^{n}$ and $b=\left(b_{i}\right)_{i} \in \mathscr{B}^{n}$ are $n$-tuples of algebra elements. The length of $E$ is defined to be the smallest number of multiplication terms required for any representation $\sum_{j} a_{j} x b_{j}$ for $E$. The case of the elementary operator is restricted to the operator $\delta_{A, B} \in B[B(\mathscr{H})]$, which is well known, as generalized derivation induced by fixed 
operators $A$ and $B$ in $B(\mathscr{H})$ and defined by $\delta_{A, B}(X)=A X-X B$.

Anderson [5] proved that if $A$ and $B$ are normal operators then

for all $X, S \in B(\mathscr{H}): S \in \operatorname{ker} \delta_{A, B} \Longrightarrow\left\|\delta_{A, B}(X)+S\right\| \geq\|S\|$,

where $\operatorname{ker} \delta_{A, B}$ denotes the kernel of $\delta_{A, B}$.

This means that the kernel of $\delta_{A, B}$ is orthogonal to its range.

This result has been generalized in different directions, to nonnormal operators (see $[6,7])$, to $C_{p}(\mathscr{H})(1 \leq p<\infty)$, and to some elementary operators (see $[8,9]$ ).

The main purpose of this paper is to characterize the elements that are orthogonal to the range of arbitrary elementary operators defined on $C_{1}(\mathscr{H})$ in nonsmoothness case and give a counter example to the results of S. Mecheri and M. Bounkhel [10].

In addition to the notations and the definitions already introduced, we set, if $\mathscr{X}$ is a normed linear space over a field $\mathbb{K}=\mathbb{R}$ or $\mathbb{C}$, we denote by $B(\mathscr{X})$ the space of all linear bounded operator on $\mathscr{X}$, the closure of the range of an operator $T \in B(\mathscr{X})$ will be denoted by $\overline{\operatorname{ran}}(T)$, the restriction of $T$ to an invariant subspace $M$ will be denoted by $T_{\mid M}$, and the commutator $A B-B A$ of the operators $A, B$ will be denoted by $[A, B]$.

\section{Main Results}

Let $\mathscr{X}$ be a normed linear space over the field $\mathbb{K}$ and $\mathscr{X}^{\dagger}$ its topological dual. Let $D$ be the (multivalued) mapping defined from $\mathscr{X}$ to $\mathscr{X}^{\dagger}$ as

$$
\forall x \in \mathscr{X}: D(x)=\left\{\varphi \in \mathscr{X}^{\dagger}: \varphi(x)=\|x\|^{2} ;\|\varphi\|=\|x\|\right\},
$$

called the normalized duality mapping. Hahn-Banach's theorem ensures that there always exists at least one support functional (a support functional $\psi$ at $x \in \mathscr{X}$ is a norm-one linear functional in $\mathscr{X}^{\dagger}$ such that $\left.\psi(x)=\|x\|\right)$ at each vector $x \in \mathscr{X}$ and therefore $D(x)$ is nonempty for every $x \in \mathscr{X}$. Moreover, it is well known that $D(x)$ is convex and weak*compact subset of $\mathscr{X}^{\dagger} . D$ is not linear in general but it is homogeneous, i.e., $\forall \alpha \in \mathbb{R}: D(\alpha x)=\alpha D(x)$.

Theorem 1. Let $K$ be a closed linear subset of $\mathscr{X}$ and $x \notin K$; then,

$$
x \perp K \Longleftrightarrow \exists \widetilde{\varphi} \in D(x): K \subseteq \operatorname{ker} \tilde{\varphi} .
$$

Proof. Let $\varphi \in D(x)$ such that $\langle\varphi, y\rangle=0$, for all $y \in K$. Then,

$$
\begin{aligned}
\varphi(x+y) & =\varphi(x)=\|x\|^{2}, \\
\|x\|^{2} & \leq\|\varphi\|\|x+y\| \leq\|x\|\|x+y\|,
\end{aligned}
$$

that is, $\|x\| \leq\|x+y\|$, for all $y \in K$. Hence, $x \perp K$.

Conversely, let $x \notin K$ such that $x \perp K$. Then, for all $y \in K$, $x$ and $y$ are linearly independent vectors. Let $L$ be the closed subspace spaned by $K$ and $\{x\}, L=[K,\{x\}]$, and define the function $\varphi$ on $L$ by $\varphi(\alpha x+\beta y)=\alpha\|x\|^{2}$ for all $y \in K$ and all $\alpha, \beta \in \mathbb{C}$. Clearly $\varphi$ is linear (by the assumption that $K$ is a linear subset of $\mathscr{X}$ ). To prove the continuity of $\varphi$, let $z \in L$, then $z=\alpha x+\beta y$ and $\varphi(z)=\alpha\|x\|^{2}$. By the definition of $\perp$ and the assumption that $x \perp K$, we derive that $\|z\| \geq\|\alpha x\|$. If $\alpha \neq 0$, we have

$$
|\varphi(z)|=|\alpha|\|x\|^{2} \cdot \frac{\|z\|}{\|z\|} \leq \frac{|\alpha|\|x\|^{2}}{\|\alpha x\|}\|z\|=\|x\|\|z\| .
$$

If $\alpha=0$, then $|\varphi(z)|=|\varphi(\beta y)|=0$. Hence,

$$
|\varphi(z)| \leq\|x\|\|z\|, \quad \text { for all } z \in L \text {. }
$$

Therefore, $\varphi$ is continuous on $L$ and $\|\varphi\|=\|x\|$. By Hahn-Banach theorem, there is a continuous linear functional $\widetilde{\varphi}$ on $\mathscr{X}$ such that $\widetilde{\varphi}_{\mid L}=\varphi$ and $\|\widetilde{\varphi}\|=\|\varphi\|$, where $\widetilde{\varphi}_{\mid L}$ is the restriction of $\widetilde{\varphi}$ on $L$. It follows by the definition of $\varphi$ and $\widetilde{\varphi}_{\mid L}=\varphi$, that $K \subseteq \operatorname{ker} \widetilde{\varphi}$ and $\tilde{\varphi} \in D(x)$.

Notation 1. Let $K$ be a nonempty subset of $\mathscr{X}$ and $T \in B(\mathscr{X})$, and we denote the duality adjoint of $T$ by $T^{\dagger}$ and set $K^{\perp^{r}}=\{x \in \mathscr{X}: x \perp y ; \forall y \in K\}$.

Remark 1. It is clear that if $\left\{x_{n}\right\}_{n}$ is a sequence in a subset $K$ converging to $y$ and $x \perp x_{n}$, for all $n$, then $x \perp y$. Hence, $x \perp K \Longrightarrow x \perp \bar{K}$.

\section{Corollary 1}
(i) $\forall x, y \in \mathscr{X}, x \perp y \Longleftrightarrow \exists \varphi \in D(x): \varphi(y)=0$
(ii) $\forall x \in \mathscr{X}, \forall \varphi \in D(x): x \perp \operatorname{ker} \varphi$
(iii) $s \perp \operatorname{ran} T \Longleftrightarrow \exists \varphi \in D(s): \varphi \in \operatorname{ker} T^{\dagger}$

Proof (i) and (ii) follow immediately from the previous Theorem 1.

(iii) By Theorem 1 and Remark $1, s \perp \operatorname{ran} T$ is equivalent to the existence of $\varphi \in D(s)$ such that $\operatorname{ran} T \subseteq \operatorname{ker} \varphi$, that is, $\varphi(T x)=\left(T^{\dagger} \varphi\right) x=0, \forall x \in \mathscr{X}$. Hence,

$$
s \perp \operatorname{ran} T \Longleftrightarrow \exists \varphi \in D(s): \varphi \in \operatorname{ker} T^{\dagger} .
$$

It is well known that $C_{1}(\mathscr{H})$ not reflexive, even not smooth and its dual $C_{1}(\mathscr{H})^{\dagger}$ is isometrically isomorphic to $B(\mathscr{H})$. This isomorphism is given by

$$
\phi: B(\mathscr{H}) \longrightarrow C_{1}(\mathscr{H})^{\dagger}, R \mapsto \phi_{R}: \phi_{R}(X)=\operatorname{tr}(X R) .
$$

Then,

$$
\begin{aligned}
\varphi \in C_{1}(\mathscr{H})^{\dagger} & \Longleftrightarrow \exists R \in B(\mathscr{H}): \phi_{R}=\varphi, \\
\|\varphi\| & =\|R\| \text { and } \varphi(X)=\operatorname{tr} R X, \quad \forall X \in C_{1}(\mathscr{H}) .
\end{aligned}
$$

Let $\xi$ be the elementary operator defined by $\xi(X)=\sum_{i=1, \ldots, n} A_{i} X B_{i} \quad$ on $\quad C_{1}(\mathscr{H})$, where $A=\left(A_{1}, A_{2}, \ldots, A_{n}\right)$ and $B=\left(B_{1}, B_{2}, \ldots, B_{n}\right)$ are $n$-tuples in $B(\mathscr{H})^{n}$. By isomorphism (11), we can assert that the duality of $\xi$ on $C_{1}(\mathscr{H})$ has the form $\xi^{\dagger}(X)=\sum_{i=1, \ldots, n} B_{i} X A_{i}$. Indeed, let $X \in C_{1}(\mathscr{H})$ and $R \in B(\mathscr{H})$; then, 


$$
\begin{aligned}
\phi_{R}(\xi(X)) & =\operatorname{tr}\left(\sum_{i=1,2, \ldots, n} A_{i} X B_{i} R\right) \\
& =\operatorname{tr}\left(X \sum_{i=1,2, \ldots, n} B_{i} R A_{i}\right) \\
& =\operatorname{tr}\left(X \xi^{\dagger}(R)\right) \\
& =\phi_{\xi^{\dagger}(R)}(X) .
\end{aligned}
$$

Let $K$ be a closed linear subspace of a hilbert space $\mathscr{H}$, and $P_{k}$ be the orthogonal projection on $K$. For a bounded operator $T$ on $\mathscr{H}$, the compression of $T$ to $K$ is the restriction $K$ to the operator $P_{k} T$. We denote by $\sigma_{p}(T)$ for the point spectrum (set of eigenvalues of $T$ ) and by $\sigma_{\mathrm{pr}}$ for the reduced point spectrum of $T$, i.e.,

$$
\sigma_{\mathrm{pr}}(T)=\left\{\lambda: T x=\lambda x \text { and } T^{*} x=\bar{\lambda} x ; x \in \mathscr{H} \backslash\{0\}\right\} .
$$

Proposition 1. Let $T \in B(\mathscr{H})$ and $\mathscr{J}$ be a two-sided ideal of $B(\mathscr{H})$.

If $\operatorname{ker} \delta_{T} \cap \mathscr{J} \subseteq \operatorname{ker} \widetilde{\delta} \cap \mathscr{J}$, then

$$
\operatorname{ker} \delta_{T} \cap \mathscr{J} \neq\{0\} \Longleftrightarrow \sigma_{p}(T) \cap \sigma_{p}\left(T^{*}\right)^{*}=\sigma_{\mathrm{pr}}(T) \text { and } \sigma_{\mathrm{pr}}(T) \neq \varnothing \text {, }
$$

where $\delta_{T}=\delta_{T, T}$.

Proof. $(\Longrightarrow)$

(1) Suppose that $0 \neq S \in \operatorname{ker} \delta_{T} \cap \mathcal{F}$, then $T S=S T$ and thus $T^{*} S=S T^{*}$ or $T S^{*}=S^{*} T$. Hence, $T|S|=|S| T$, where $|S|$ is a normal compact operator. Since $\sigma(|S|) \neq\{0\}$, then there is $0 \neq \lambda \in \sigma(|S|)$ such that the Riesz projector $P_{\lambda}$ is of finite rank and $P_{\lambda} \in T^{0}$ (the bicommutant of $T$ ). It follows from $S \in \operatorname{ker} \delta_{T} \subseteq$ $\operatorname{ker} \widetilde{\delta}_{T}$ that $P_{\lambda} \in \operatorname{ker} \delta_{T} \subseteq \operatorname{ker} \widetilde{\delta}_{T}$ and therefore the subspace $\operatorname{ran} P_{\lambda}$ reduces $T$ and $m \operatorname{ran} P_{\lambda}<\infty$. Let

$$
\mathscr{J}_{\lambda}=\left\{\left.P_{\lambda} S\right|_{\operatorname{ran} P_{\lambda}}: S \in \mathscr{J}\right\}
$$

where $\left.P_{\lambda} S\right|_{\operatorname{ran} P_{\lambda}}$ is the compression of $S$ on $\operatorname{ran} P_{\lambda}$ so that

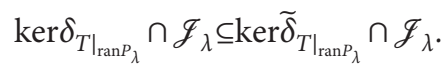

Indeed, let us consider the decomposition $\mathscr{H}=\operatorname{ran} P_{\lambda} \oplus\left(\operatorname{ran} P_{\lambda}\right)^{\perp}$, then $T$ can be written $T=T_{1} \oplus T_{2}$, where $T_{1}=\left.T\right|_{\operatorname{ran} P_{\lambda}}$.

Let $S_{\lambda} \in \operatorname{ker} \delta_{\left.T\right|_{\text {ran }}} \cap \mathscr{F}_{\lambda}$, then $T_{1} S_{\lambda}=S_{\lambda} T_{1}$. Choosing $S=S_{\lambda} \oplus 0$ implies $S \in \operatorname{ker} \delta_{T} \cap \mathscr{J} \subseteq \operatorname{ker} \widetilde{\delta}_{T} \cap \mathscr{J}$. Thus, $T S^{*}=S^{*} T$ and therefore $T_{1} S_{\lambda}^{*}=S_{\lambda}^{*} T_{1}$.

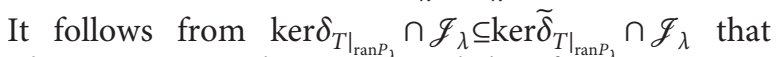
$\left.T\right|_{\text {ran } P_{\lambda}}$ is a normal operator and therefore

$$
\sigma_{\operatorname{pr}}\left(\left.T\right|_{\operatorname{ran} P_{\lambda}}\right)=\sigma_{p}\left(\left.T\right|_{\operatorname{ran} P_{\lambda}}\right)=\sigma\left(\left.T\right|_{\operatorname{ran} P_{\lambda}}\right)
$$

which implies that $\sigma_{\mathrm{pr}}(T) \subseteq \sigma_{p}(T) \cap \sigma_{p}\left(T^{*}\right)^{*}$ and $\sigma_{\mathrm{pr}}(T)$ is not an emptyset.

(2) Let $\lambda \in \sigma_{p}(T) \cap \sigma_{p}\left(T^{*}\right)^{*}$, then there exist $x, y \in \mathscr{H} \backslash\{0\}$ such that $T x=\lambda x$ and $T^{*} y=\bar{\lambda} y$. Hence, there is $S$ such that

$$
S=x \otimes y \in \operatorname{ker} \delta_{T} \cap \mathscr{J} \subseteq \operatorname{ker} \widetilde{T} \cap \mathscr{J},
$$

Applying Fuglede-Putnam property [6], we get that $\overline{\operatorname{ran} S}$ reduces $T$ and $\left.T\right|_{\overline{\mathrm{ran}} S}$ is a normal operator. Since $x \in \operatorname{ker}\left(\left.T\right|_{\overline{\operatorname{ran}} S}-\lambda\right)$, we derive that $T^{*} x=\bar{\lambda} x$ and therefore $\lambda \in \sigma_{\mathrm{pr}}(T)$. The inclusion is proved.

Let us prove the reverse implication $(\Leftarrow)$.

Let $\lambda \in \sigma_{p}(T) \cap \sigma_{p}\left(T^{*}\right)^{*}$, then there exist $x, y \in \mathscr{H} \backslash\{0\}$ such that $T x=\lambda x$ and $T^{*} y=\bar{\lambda} y$. Setting $S=x \otimes y$, then $S \in \operatorname{ker} \delta_{T} \cap \mathcal{J}$.

Corollary 2. Let $A$ be a normal operator in $B(\mathscr{H})$ with $\sigma_{p r}(A)=\varnothing$ and $S \notin \operatorname{ker} \delta_{A}$ be a positive operator in $C_{1}(\mathscr{H})$,

$$
\left\|\delta_{A}(X)+S\right\|_{1} \geq\|S\|_{1}, \quad \text { for all } X \in C_{1}(\mathscr{H})
$$

Proof. Since $S$ is positive, then $S=|S|$, hence $\operatorname{tr}\|S\|_{1} S=\operatorname{tr}\|S\|_{1}|S|=\|S\|^{2}$ so that there is $T=\|S\|_{1} I \in \operatorname{ker} \delta_{A}$, and therefore by Corollary 1, isomorphisms (11) and (13), we get $S$ is orthogonal to $\operatorname{ran} \delta_{A}$ i.e.,

$$
\left\|\delta_{A}(X)+S\right\|_{1} \geq\|S\|_{1}, \quad \text { for all } X \in C_{1}(\mathscr{H}) .
$$

However, $\delta_{A}$ is a normal derivation satisfying $\operatorname{ker} \delta_{A} \subseteq \operatorname{ker} \widetilde{\delta}_{A}$ and by the previous proposition, $\operatorname{ker} \delta \cap C_{1}(\mathscr{H})=\{0\}$, and this means that $S \notin \operatorname{ker} \delta_{A}$.

We recall that the characterization of the points in $C_{1}(\mathscr{H})$ that are orthogonal to the range of the elementary operators has been carried out by certain authors for smooth case and for certain kind of elementary operators $[9,11]$. In nonsmoothness case, S. Mecheri and M. Bounkhel [10] have obtained the following result.

Proposition 2 (see [10]). Let $A, B$ be normal operators in $B(\mathscr{H})$ and let $S=U|S| \in C_{1}(\mathscr{H})$. Then, $S \in \operatorname{ker} \delta_{A, B}$ if and only if

$$
\left\|d_{A, B}(X)+S\right\|_{1} \geq\|S\|_{1}, \quad \forall X \in C_{1}(\mathscr{H}),
$$

where $d_{A, B}=\Delta_{A, B}$ or $d_{A, B}=\delta_{A, B}$ for $\Delta_{A, B}(X)=A X B-X$ and $\delta_{A, B}(X)=A X-X B$.

Remark 2

(1) The previous result shows that $\left(\operatorname{ran} d_{A, B}\right)^{\perp}$ $\cap C_{1}(c H)=\operatorname{ker} d_{A, B} \cap C_{1}(\mathscr{H})$. In what follows, we show that this is not true, i.e., $\left(\operatorname{ran} \delta_{A}\right)^{\perp} \cap$ 
$C_{1}(\mathscr{H}) \nsubseteq \operatorname{ker} \delta_{A} \cap C_{1}(c H)$. Indeed, let $\mathscr{H}=l^{2}(\mathbb{Z})$, $A \in B(H): A e_{n+1}=e_{n} ; n \in \mathbb{Z}$, and $S=e_{1} \otimes e_{1}$, where $\left\{e_{n}: n \in \mathbb{Z}\right\}$ is an orthonormal basis for $\mathscr{H}$. Hence, $A$ is a unitary operator (bilateral shift) with $\sigma_{\mathrm{pr}}(A)=\varnothing$ and $S \in C_{1}(\mathscr{H})$. By simple calculus, we obtain

$$
\begin{aligned}
A\left(e_{1} \otimes e_{1}\right)-\left(e_{1} \otimes e_{1}\right) A & =e_{0} \otimes e_{1}-e_{1} \otimes A^{*} e_{1} \\
& =e_{0} \otimes e_{1}-e_{1} \otimes e_{2} \neq 0 .
\end{aligned}
$$

Thus, $S \notin \operatorname{ker} \delta_{A}$.

On the contrary, $\phi_{I}(S)=\operatorname{tr}\left(e_{1} \otimes e_{1}\right)=1 \quad$ and $\left\|\phi_{I}\right\|=\|I\|=1$, where $I$ is the identity operator on $\mathscr{H}$. Thus, $\phi_{I} \in D(S)$. Since $I \in \operatorname{ker} \delta_{A}$, we get by (13), $\phi_{I} \in \operatorname{ker} \delta_{A}^{\dagger}$ so that it follows from Corollary 1 that $S \perp \operatorname{ran} \delta_{A}$.

(2) Furthermore, Corollary 2 provides a class of operators as counterexample to the orthogonal decomposition $C_{1}(\mathscr{H})=\overline{\operatorname{ran}} \delta_{A} \oplus \operatorname{ker} \delta_{A}$ so that the problem of characterization of operators in $C_{1}(\mathscr{H})$ that are orthogonal to the range of elementary operators is still open.

In this note, as an application of the previous section, we shall study this problem in different directions for arbitrary elementary operators.

Theorem 2. Let $\xi$ be the elementary operator defined on $C_{1}(\mathscr{H})$ by

$\xi(X)=\sum_{i=1, \ldots, n} A_{i} X B_{i}$, where $A=\left(A_{1}, \ldots, A_{n}\right)$ and $B=$ $\left(B_{1}, \ldots, B_{n}\right)$ are $n$-tuples of operators in $B(\mathscr{H})^{n}$ and let $S \in C_{1}(\mathscr{H})$ with the condition $\operatorname{ker} \xi=\operatorname{ker} \widetilde{\xi}$. Let $R=U|R|=S$ or $S^{*}$, then

(1) If $\operatorname{ker} R$ is invariant under $R^{*}, A_{i}$ and $B_{i}^{*}$ for all $i=1, \ldots, n$, then there holds

$\forall X \in C_{1}(\mathscr{H}):\|\xi(X)+R\|_{1} \geq\|R\|_{1} \Longrightarrow \xi(U)=0$.

If $\operatorname{ker} R$ reduces $R, A_{i}$ and $B_{i}^{*}$ for all $i=1, \ldots, n$, then there holds

$\xi(U)=0 \Longleftrightarrow \forall X \in C_{1}(\mathscr{H}):\|\xi(X)+R\|_{1} \geq\|R\|_{1}$.

(2) If ker $R$ is invariant under $R^{*}, A_{i}$ and $\left[|\operatorname{ran} R|, B_{i}\right]=0$ for all $i=1, \ldots, n$, then there holds

$\forall X \in C_{1}(\mathscr{H}):\|\xi(X)+R\|_{1} \geq\|R\|_{1} \Longrightarrow \xi(R)=0$.

If $\operatorname{ker} R$ reduces $R, A_{i}$ and $\left[|R|, B_{i}\right]=0$ for all $i=1, \ldots, n$, then there holds

$$
\xi(R)=0 \Longleftrightarrow \forall X \in C_{1}(\mathscr{H}):\|\xi(X)+R\|_{1} \geq\|R\|_{1} .
$$

Proof

(1) We prove only the case when $R=S=U|S|$, the case $R=S^{*}$ is similar to the previous one.

If kerS is invariant under $S^{*}, A_{i}$ and $B_{i}^{*}$ for all $n=1, \ldots, n$, then with respect to the decomposition $\mathscr{H}=(\operatorname{ker} S)^{\perp} \oplus \operatorname{ker} S$, we get the following $S=S_{1} \oplus 0, U=U_{1} \oplus 0$ and

$$
\begin{aligned}
A_{i} & =\left[\begin{array}{cc}
A_{i}^{1} & A_{i}^{2} \\
0 & A_{i}^{3}
\end{array}\right], \\
B_{i} & =\left[\begin{array}{cc}
B_{i}^{1} & 0 \\
B_{i}^{2} & B_{i}^{3}
\end{array}\right], \\
1 & \leq i \leq n .
\end{aligned}
$$

It is clear that

$X=\left[\begin{array}{cc}X_{1} & 0 \\ 0 & 0\end{array}\right] \in C_{1}(\mathscr{H}) \Longrightarrow X_{1} \in C_{1}\left((\operatorname{ker} S)^{\perp}\right)$

and $\xi(X)=\xi_{1}\left(X_{1}\right) \oplus 0$, where $\xi_{1}\left(X_{1}\right)=\sum_{i=1, \ldots, n} A_{i}^{1} X_{1}$ $B_{i}^{1}$. Hence,

$$
\begin{aligned}
\forall X \in C_{1}(\mathscr{H}):\|\xi(X)+S\|_{1} & \geq\|S\|_{1} \Longrightarrow\left\|\xi_{1}\left(X_{1}\right)+S_{1}\right\|_{1} \\
& \geq\left\|S_{1}\right\|_{1}, \forall X_{1} \in C_{1}\left((\operatorname{ker} S)^{\perp}\right) .
\end{aligned}
$$

Since $S_{1}$ is injective and $S_{1}=U_{1}\left|S_{1}\right|$, then $S_{1}$ is a smooth point in $C_{1}\left((\operatorname{ker} S)^{\perp}\right)$. So, it follows by Corollary 1 and the uniqueness of $T$ that $T=\left\|S_{1}\right\|_{1} U_{1}^{*}$ and then by ismorphism (11), $\sum_{i=1, \ldots, n} B_{i}^{1} U_{1}^{*} A_{i}^{1}=0=\sum_{i=1, \ldots, n} A_{i}^{*} U_{1} B_{i}^{1}$.

From the hypothesis $\operatorname{ker} \xi=\operatorname{ker} \tilde{\xi}$, we derive that $\operatorname{ker} \xi_{1}=$ $\operatorname{ker} \widetilde{\xi}_{1}$ and then $\sum_{i=1, \ldots, n} A_{i}^{1} U_{1} B_{i}^{1}=0$; therefore, $\xi(U)=$ $\sum_{i=1, \ldots, n} A_{i} U B_{i}=0$ which means that $U \in \operatorname{ker} \xi$.

For the reverse implication, if $\xi(\underset{U}{U})=0$, then $\xi_{1}\left(U_{1}\right)=0$, and since $\operatorname{ker} \xi_{1}=\operatorname{ker} \widetilde{\xi}_{1}$, we get $\widetilde{\xi}_{1}\left(U_{1}\right)=0=\xi_{1}^{\dagger}\left(U_{1}^{*}\right)$. We derive from Corollary 1 that

$$
\left\|\xi_{1}\left(X_{1}\right)+S_{1}\right\|_{1} \geq\left\|S_{1}\right\|_{1} ; \quad \forall C_{1}\left((\operatorname{ker} S)^{\perp}\right)
$$

and since $\operatorname{ker} S$ reduces both $A_{i}$ and $B_{i}$ for all $i=1, \ldots, n$, we obtain, for $X=\left(X_{k}\right)_{k=1, \ldots, 4} \in C_{1}(\mathscr{H})$ and $A_{i}=A_{i}^{1} \oplus A_{i}^{2}, B_{i}=B_{i}^{1} \oplus B_{i}^{2}$, that

$$
\begin{aligned}
\|\xi(X)+S\|_{1} & =\left\|\left[\begin{array}{cc}
\xi_{1}\left(X_{1}\right)+S_{1} & * \\
* & *
\end{array}\right]\right\|_{1} \geq\left\|\xi_{1}\left(X_{1}\right)+S_{1}\right\|_{1} \\
& \geq\left\|S_{1}\right\|_{1}=\|S\|_{1} .
\end{aligned}
$$


(2) If $\operatorname{ker} S$ is invariant under $S^{*}, A_{i}$ and $\left[|S|, B_{i}\right]=0$ for all $i=1, \ldots, n$, then kerS reduces $B$, following the same steps as in the previous case, we get $\sum_{i=1, \ldots, n} A_{i} U B_{i}=0$. Hence, $\quad\left[|S|, B_{i}\right]=0$ for all $i=1, \ldots, n$ implying $\xi(S)=0$. Since $\left|S_{1}\right|$ is injective, then $\xi\left(U_{1}\right)=0$. We argue similarly as in the previous case to finish the proof.

Remark 3. If $S$ is a smooth point in $C_{1}(\mathscr{H})$, then $\operatorname{ker} S$ or $\operatorname{ker} S^{*}$ are trivial, then relations (11) and (13) hold for any elementary $\xi$ satisfying $\operatorname{ker} \xi=\operatorname{ker} \tilde{\xi}$, and relation (13) hold under the conditions $\left[|S|, B_{i}\right]=0$ for sll $i=1, \ldots, n$ for any operator $\xi(X)=\sum_{i=1, \ldots, n} A_{i} X B_{i}$ with $\operatorname{ker} \xi=\operatorname{ker} \tilde{\xi}$.

Lemma 1. Let $A=\left(A_{1}, \ldots, A_{n}\right), B=\left(B_{1}, \ldots, B_{n}\right)$ with $A_{i}$ and $B_{i}$ be operators in $B(\mathscr{H})$ such that $\sum_{i} A_{i} A_{i}^{*} \leq 1, \sum_{i} A_{i}^{*} A_{i} \leq 1, \sum_{i} B_{i}^{*} B_{i} \leq 1$, and $\sum_{i} B_{i} B_{i}^{*} \leq 1$. Let $\xi$ be the elementary operator defined on $C_{p: 1 \leq p<\infty}(\mathscr{H})$ by

$$
\xi(X)=\sum_{i=1, \ldots, n} A_{i} X B_{i}-X .
$$

Then,

(i) $\operatorname{ker} \xi=\operatorname{ker} \tilde{\xi} \Longrightarrow \operatorname{ker} \xi^{\dagger}=\operatorname{ker} \tilde{\xi}^{\dagger}$

(ii) If $\xi(S)=0=\tilde{\xi}(S)$ for some compact operator $S$, then $\left[|S|, B_{i}\right]=0$ for all $1 \leq i \leq n$

Proof

(i) We have $\xi^{\dagger}(S)=0 \Longleftrightarrow \tilde{\xi}\left(S^{*}\right)$. Since $\operatorname{ker} \xi=\operatorname{ker} \tilde{\xi}$, it follows that

$$
\tilde{\xi}(S)=0 \Longleftrightarrow \xi\left(S^{*}\right)=0 \Longleftrightarrow \widetilde{\xi}^{\dagger}(S)=0 .
$$

(ii) See [11].

As an application of Theorem 2 and by using Lemma 1, we obtain the following result which generalizes that given by B. P. Duggal in the smooth case [9].

Corollary 3. Let $\xi(X)=\sum_{i=1, \ldots, n} A_{i} X B_{i}-X$ such that

$$
\begin{aligned}
& \sum_{i} A_{i} A_{i}^{*} \leq 1, \\
& \sum_{i} A_{i}^{*} A_{i} \leq 1, \\
& \sum_{i} B_{i}^{*} B_{i} \leq 1, \\
& \sum_{i} B_{i} B_{i}^{*} \leq 1,
\end{aligned}
$$

with $\operatorname{ker} \xi=\operatorname{ker} \tilde{\xi}$ and $\operatorname{ker} \xi_{B}=\operatorname{ker} \tilde{\xi}_{B}$. For all $S \in C_{1}(\mathscr{H})$, the following assertions hold:

(i) If kerS reduces $S$, then

$$
S \perp \operatorname{ran} \xi \Longrightarrow S \in \operatorname{ker} \xi
$$

(ii) If $\operatorname{ker} S$ reduces $S$ and $S \in \operatorname{ker} \xi_{B}$, then

$$
S \perp \operatorname{ran} \xi \Longrightarrow S \in \operatorname{ker} \xi
$$

In what follows, for an elementary operator $\xi$ defined on $C_{1}(\mathscr{H})$ by

$$
\xi(X)=\sum_{i=1, \ldots, n} A_{i} X B_{i},
$$

where $A=\left(A_{1}, \ldots, A_{n}\right)$ and $B=\left(B_{1}, \ldots, B_{n}\right)$ are $n$-tuples of operators in $B(\mathscr{H})^{n}$, we shall prove that there are operators, not necessary in the kernel, which are orthogonal to the range without the condition $\operatorname{ker} \xi \subseteq \operatorname{ker} \tilde{\xi}$.

Recall that if $\mathscr{X}$ is a Banach space, then the function $\psi_{x, y}$ defined by

$$
\psi_{x, y}(t)=\frac{\|x+t y\|-\|x\|}{t}
$$

is increasing on $(0, \infty)$, convex for all $x, y \in \mathscr{X}$, and $\lim _{t \rightarrow 0^{+}} \psi_{x, y}(t)$ exists for all $x, y \in \mathscr{X}$.

So, from the properties of $\psi_{x, y}$ and the fact that $\lim _{t \longrightarrow 0^{+}} \psi_{x, y}(t)=\lim _{t \longrightarrow 0^{-}} \psi_{x,-y}(t)$, it follows that $x \perp_{B-J} y \Longleftrightarrow \lim _{t \longrightarrow 0^{-}} \psi_{x, y}(t) \leq 0 \leq \lim _{t \longrightarrow 0^{+}} \psi_{x, y}(t), \quad \forall t>0$.

Indeed, if $x \perp_{B-I} y$, then $\|x+\lambda y\|-\|x\| \geq 0$, for all $\lambda \in \mathbb{C}$. Therefore, by setting $\lambda=t e^{i \theta}$, where $t=|\lambda|$ and $\theta \in \mathbb{R}$ and since $\psi_{x, y}$ is increasing on $(0, \infty)$, then $0 \leq \lim _{t \longrightarrow 0^{+}} \psi_{x, y}(t)$. The converse holds from the convexity of $\psi_{x, y}$.

Lemma 2. Let $S, T \in C_{p}(1 \leq p<\infty)$ and $\mathscr{K}$ be a closed subspace of $\mathscr{H}$. If $\mathscr{K}$ reduces $S$, then

$$
\left.S\right|_{\mathscr{K}} \perp_{B-J} T_{11} \text { and }\left.S\right|_{\mathscr{K}^{\perp}} \perp_{B-J} T_{22} \Longrightarrow S \perp_{B-J} T \text {, }
$$

where $T_{11}$ and $T_{22}$ are the compression of $T$ to the subsapces $\mathscr{K}$ and $\mathscr{K}^{\perp}$, respectively.

Proof. With respect to the decomposition $\mathscr{H}=\mathscr{K} \oplus \mathscr{K}^{\perp}$, we can write $S=\left.\left.S\right|_{\mathscr{K}} \oplus S\right|_{\mathscr{K}^{\perp}}$ and $T=\left(T_{i j}\right)_{i, j=1,2}$, then

$$
S+\lambda T=\left[\begin{array}{cc}
\left.S\right|_{c K}+\lambda T_{11} & \lambda T_{12} \\
\lambda T_{21} & \left.S\right|_{\mathscr{K}^{\perp}}+\lambda T_{22}
\end{array}\right], \quad \forall \lambda \in \mathbb{C} .
$$

Since the $p$-norm od an operator matrix always dominates the $p$-norm of its diagonal parts [12], then

$$
\begin{aligned}
\|S+\lambda T\|_{p} & \geq\left\|\left.S\right|_{\mathscr{K}}+\lambda T_{11}\right\|_{p}+\left\|\left.S\right|_{\mathscr{K}^{\perp}}+\lambda T_{22}\right\|_{p} \\
\|S\|_{p} & =\left\|\left.S\right|_{\mathscr{K}}\right\|_{p}+\left\|\left.S\right|_{\mathscr{K}^{\perp}}\right\|_{p^{p}}
\end{aligned}
$$

Setting $\lambda=t e^{i \theta}$, where $t=|\lambda|$ and $\theta \in \mathbb{R}$, then

$$
\begin{aligned}
\left\|S+t e^{i \theta} T\right\|_{p}-\|S\|_{p} \leq & \left\|\left.S\right|_{\mathscr{K}}+t e^{i \theta} T_{11}\right\|_{p}-\left\|\left.S\right|_{\mathscr{K}}\right\|_{p} \\
& +\left\|\left.S\right|_{\mathscr{K}^{\perp}}+t e^{i \theta} T_{22}\right\|_{p}-\left\|\left.S\right|_{\mathscr{K}^{\perp}}\right\|_{p} .
\end{aligned}
$$

Thus, 


$$
\lim _{t \longrightarrow 0^{+}} \psi_{S, e^{i \theta} T}(t) \leq\left.\lim _{t \longrightarrow 0^{+}} \psi_{S}\right|_{\mathscr{K}}, e^{i \theta} T_{11}(t)+\lim _{t \longrightarrow 0^{+}} \psi_{\left.S\right|_{\mathscr{K}}, e^{i \theta} T_{22}}(t) .
$$

So, the result follows from (40).

Proposition 3. Let $\xi$ be the elementary operator defined on $C_{1}(\mathscr{H})$ by $\xi=L_{A} R_{B}$, where $A=\left(A_{1}, \ldots, A_{n}\right), B=$ $\left(B_{1}, \ldots, B_{n}\right)$ are $n$-tuples of operators in $B(\mathscr{H})$. If $S=U|S|$ such that $\operatorname{ker} S$ is invariant under $S^{*}$ and $A_{i}$ for all $i=1, \ldots, n$ (resp. kerS* is invariant under $S$ and $A_{i}$ for all $i=1, \ldots, n$ ), then

$$
\begin{aligned}
L_{B_{11}} R_{A_{11}}\left(U_{1}^{*}\right) & =0 \Longrightarrow S \perp \text { ran } \xi \\
\left(\operatorname{resp} . L_{B_{11}} R_{A_{11}}\left(U_{1}\right)\right. & =0 \Longrightarrow S \perp \text { ran } \xi),
\end{aligned}
$$

such that $A_{11}=\left(A_{11}^{1}, A_{11}^{2}, \ldots, A_{11}^{n}\right) ; A_{k}=\left(A_{i, j}\right)_{i, j=1,2}$, $k=1, \ldots, n$ (with the same notations for $B$ ), where $A_{11}^{k}\left(\right.$ resp. $\left.B_{11}^{k}\right)$ is the compression of $A_{k}\left(\right.$ resp. $\left.B_{k}\right)$ to $(\mathrm{ker} S)^{\perp}$ and $S_{1}=U_{1}\left|S_{1}\right|=\left.S\right|_{\left.(\mathrm{ker} S)^{\perp}\right)}$.

Proof. Let $\mathscr{H}=(\operatorname{ker} S)^{\perp} \oplus \operatorname{ker} S$; since kerS is invariant under $S^{*}$ and $A_{i}$ for all $i=1, \ldots, n$, we obtain

$$
\begin{aligned}
& S=S_{1} \oplus 0, \quad A_{k}=\left[\begin{array}{cc}
A_{11}^{k} & 0 \\
A_{21}^{k} & A_{22}^{k}
\end{array}\right], \\
& B_{k}=\left(B_{i, j}^{k}\right)_{i, j=1,2},
\end{aligned}
$$

for all $k=1, \ldots, n$. Let $X=\left(X_{i, j}\right)_{i, j=1,2} \in C_{1}(\mathscr{H})$, by simple calculation, we obtain that the compression of $\xi(X)$ to the subspace $(\operatorname{ker} S)^{\perp}$ is

$$
\xi_{11}\left(X_{11}\right)=\sum_{k=1}^{n} A_{11}^{k} X_{11} B_{11}^{k}=L_{A_{11}} R_{B_{11}}\left(X_{11}\right),
$$

so that it follows from the previous lemma that if $S_{1} \perp \operatorname{ran} \xi_{11}$, then $S \perp \operatorname{ran} \xi$. Since $S_{1}$ is a smooth point (injective operator) in $C_{1}\left((\operatorname{ker} S)^{\perp}\right)$ and $U_{1}\left|S_{1}\right|$ its polar decomposition, where $U_{1}$ is an isometry in $B\left((\operatorname{ker} S)^{\perp}\right)$. Applying Corollary 3 (ii), we get that if $L_{B_{11}} R_{A_{11}}\left(U_{1}^{*}\right)=0$, then $S \perp$ ran $\xi$.

Example 1. Let $\mathscr{H}=l_{2}(\mathbb{Z})$ and $A e_{n+1}=e_{n} n \in \mathbb{Z}$ and

$$
S e_{n+1}= \begin{cases}\frac{1}{2^{n+1}} e_{n}, & \text { if } n \geq 0, \\ 0, & \text { if } n \leq-1 .\end{cases}
$$

We can assert that

$$
S^{*} e_{n}= \begin{cases}\frac{1}{2^{n+1}} e_{n+1}, & \text { if } n \geq 0, \\ 0 & \text { if } n \leq-1 .\end{cases}
$$

$A$ is unitary with $\sigma_{\mathrm{pr}}(A)=\varnothing($ see Remark 2), then $\operatorname{ker} \delta_{A} \cap C_{1}(\mathscr{H})=\{0\}$. It is clear that $\operatorname{ker} S$ is spanned by the set $\left\{\cdots, e_{-2}, e_{-1}, e_{0}\right\}$ and $\operatorname{ker} S^{*}$ is spanned by the set $\left\{\cdots, e_{-2}, e_{-1}\right\}$, then $\operatorname{ker} S^{*} \subset \operatorname{ker} S$ so that $\operatorname{ker} S^{*}$ reduces $S^{*}$, also $A\left(\operatorname{ker} S^{*}\right) \subseteq \operatorname{ker} S^{*}$. Hence, $S=\left.S\right|_{\left(\operatorname{ker}^{*}\right)^{\perp}} \oplus 0$. If $S=U|S|$ and $U_{1}=\left.U\right|_{\left(\mathrm{kerS}^{*}\right)^{\perp}}$, then

$$
\begin{gathered}
U e_{n}= \begin{cases}e_{n-1}, & \text { if } n \geq 1, \\
0 & \text { if } n \leq 0,\end{cases} \\
U_{1} e_{n}= \begin{cases}e_{n-1}, & \text { if } n \geq 1, \\
0 & \text { if } n=0 .\end{cases}
\end{gathered}
$$

If $A_{1}$ is the compression of $A$ to $\left(\operatorname{ker} S^{*}\right)^{\perp}$, then $A_{1} e_{n}=\left\{\begin{array}{ll}e_{n-1}, & \text { if } n \geq 1 \\ 0 & \text { if } n \leq 0\end{array}\right.$.

We have $A_{1} U_{1} e_{n}=U_{1} A_{1} e_{n}=e_{n-2}$ for all $n \geq 2$ and $A_{1} U_{1} e_{n}=U_{1} A_{1} e_{n}=0$ for $n=0,1$. Then, $U_{1} \operatorname{ker} \delta_{A}$, and therefore by Proposition 3, we get $S \perp \operatorname{ran} \delta_{A}$. We have also $S^{*} e_{0}=1 / 2 e_{1} \notin \operatorname{ker} S$ which means that kerS is not invariant under $S^{*}$.

\section{Data Availability}

The data used to support the findings of this study are available from the corresponding author upon request.

\section{Conflicts of Interest}

The authors declare that they have no conflicts of interest.

\section{Acknowledgments}

The authors thank the anonymous referees for their helpful remarks and extend their appreciation to the Deanship of Scientific Research at King Khalid University for funding this work through General Research Project grant number (G.R.P- 93-41).

\section{References}

[1] B. Simon, "Trace ideals and their applications," London Mathematical Society Lecture Note Series, Vol. 35, Cambridge University Press, Cambridge, UK, 1979.

[2] G. Birkhoff, "Orthogonality in linear metric spaces," Duke Mathematical Journal, vol. 1, no. 2, pp. 169-172, 1935.

[3] R. C. James, "Orthogonality and linear functionals in normed linear spaces," Transactions of the American Mathematical Society, vol. 61, no. 2, p. 265, 1947.

[4] R. Harte, Skew Exactness and Range-Kernal Orthogonality, Filomat, Visegradska, Serbia, 2005.

[5] J. Anderson, "On normal derivations," Proceedings of the American Mathematical Society, vol. 38, no. 1, pp. 136-140, 1973.

[6] A. Bachir and A. Segres, "Generalized Fuglede-Putnam's theorem and orthogonality," AJMAA, vol. 1, pp. 1-5, 2004.

[7] B. P. Duggal, "Putnam-Fuglede theorem and the range-kernel orthogonality of derivations," International Journal of Mathematics and Mathematical Sciences, vol. 27, no. 9, pp. 573-582, 2001.

[8] B. P. Duggal, "Subspace gaps and range-kernel orthogonality of an elementary operator," Linear Algebra and Its Applications, vol. 383, pp. 93-106, 2004.

[9] B. P. Duggal, "Range-kernel orthogonality of the elementary operator $X \mapsto \sum_{i=1}^{n} A_{i} X B_{i}-I$." Linear Algebra and Its Applications, vol. 337, no. 1-3, pp. 79-86, 2001.

[10] S. Mecheri and M. Bounkhel, "Some variant of Anderson's inequalityin $C_{1}$ class," Journal of Inequalities in Pure and Applied Mathematics, vol. 4, no. 1, p. 24, 2003. 
[11] A. Turnšek, "Elementary operators and orthogonality," Linear Algebra and its Applications, vol. 317, no. 1-3, pp. 207-216, 2000.

[12] R. Bhatia and F. Kittaneh, "Norm inequalities for partitioned operators and an application," Mathematische Annalen, vol. 287, no. 1, pp. 719-726, 1990. 